\title{
Aufbau und Betrieb einer Station zur Messung lufffremder Stoffe
}

\author{
Prof. Dr. rer. nat. Klaus R. F. Bätjer, Iris Geike
}

\section{Einleitung}

Im Jahre 1992 wurden erste Schritte unternommen, Meßgeräte für die Technische Fachhochschule Wildau (TFH Wildau) zu erwerben, die es dieser ermöglichen sollen, entsprechend den Standards der Luftuiberwachung in den Bundesländern Berlin und Brandenburg, wichtige luftgetragene Schadstoffe kontinuierlich messend zu verfolgen. Bereits 1992 wurde fuir die Zukunft erwartet, daß in den neuen Bundesländern weiterhin alte und veraltete Industriezweige aufgeben müßten; die Industrieerneuerungen hinsichtlich der Emissionen jedoch so gering sein würden, daß es eine Perspektive für die Ausbildung der Studenten und für die Forschung in der Luftreinhaltung an der TFH Wildau nur für Immissionsuntersuchungen geben könnte. Nach wie vor ist die Verschmutzung der Atmosphäre durch zahlreiche, bislang noch nicht sanierte Emittenten aus den Bereichen Haushalt, Industrie und Verkehr erheblich, wenn auch, abgesehen von einigen Ausnahmen, abnehmend. Im Fachbereich Physikalische Technik der TFH Wildau beschäftigt sich eine von mehreren Spezialisierungsrichtungen mit der Untersuchung u. a. der Ausbreitung und des Verhaltens luftgetragener Schadstoffe. Die aufzubauende Luftmeßstation in dem Container sollte mindestens den folgenden Kriterien genuigen:

1. Der Ausbildung der Studenten an praxisnahen und problemorientierten Beispielen und Geräten zu genügen;

2. Einen kontinuierlichen Betrieb mit Datenaufnahme zu gewährleisten und damit später einen möglichen Datenaustausch mit den Bundesländern Berlin und Brandenburg zu ermöglichen; und

3. Die Möglichkeit bieten, eigenständigen Forschungen an der TFH Wildau nachgehen zu können.

Leider konnten die von der Hochschule 1992 beschafften Meßgeräte erst 1995 in dem vorhandenen Meßcontainer auf dem Gelände der TFH Wildau in der Nähe des Hauses 3 in Betrieb genommen werden. Zum Jahreswechsel 1995/1996 wurde noch eine kleine meteorologische Meßstation installiert, so daß, abgesehen von dem nicht optimalen Standort des Containers, ein vollwertiges Meßsystem der TFH Wildau zur Verfügung steht.

\section{Aufbau der Meßsysteme im Container}

1994 wurde der Container beschafft und auf dem, zum damaligen Zeitpunkt, einzig zugänglichen Gelände, suidlich vom Haus 3 in Wildau aufgestellt. Im Jahr 1995 wur-

de entsprechend den finanziellen Möglichkeiten begonnen, die verschiedenartigen Meß- und Probenahmesysteme zu installieren; dieser Erstaufbau findet seinen vorläufigen Abschluß zum Jahreswechsel 1995/1996. Der wärmeisolierte Container hat Maße von zirka $6 \mathrm{x}$ 2,5 x 2,5 Metern (m), ist durch eine Doppeltuir von der Ruickseite zu betreten und besitzt auf der einen Breitseite ein und auf der Längsseite zwei Fenster, die alle zu öffnen sind. Von außen sind Jalousien vorhanden, die in der Regel herabgelassen sind. Die Innenausstattung entspricht dem üblichen Standard eines Containerkleinlaboratoriums. Folgende Systeme wurden am und im Meßcontainer installiert:

- Datenauswertesysteme; Meß- und Probenahmesysteme für Gase und für Schwebstaub;

- Heizungs- und Klimageräte sowie ein meteorologisches Meß- und Probenahmesystem.

Trotz der durch Bäume vor der Sonnenstrahlung geschützten Lage, die den Immissionsmessungen nicht förderlich ist - eine Umsetzung des Containers ist auf mittelfristige Sicht unumgänglich -, erwärmte sich das Containerinnere im Sommer 1995 so stark, daß teilweise die Elektronik, die Meßgeräte und der Rechner, auch durch ihre eigene Wärmeproduktion, versagten.

Die Abbildung 1 zeigt den Container von der Straßenseite her, deutlich sind die äußeren Meß- und Probenahmesysteme zu erkennen.

Links neben dem Container befindet sich das Meßgerät fuir die Niederschlagsmessungen (u. a. Regen), und dort neben dem Container und über dem Dach des Containers befindet sich der Mast für das meteorologische Meßsystem, dann von links nach rechts auf dem Dach

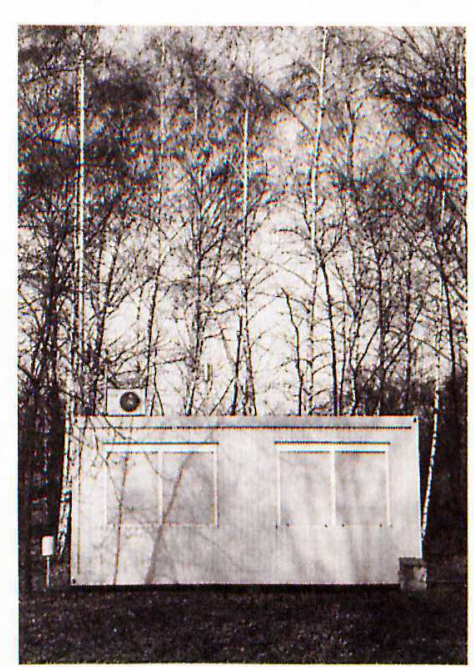

Abb. 1: Der Luftmeßcontainer der TFH Wildau mit seinen äußeren Aufbauten 1996 das Ansaug- und Probenahmerohr für die staubförmigen Anteile der Luft, das eckige Klimasystem und schließlich das Ansaug- und Probenahmerohr fuir die gasförmigen Luftschadstoffe.

An der Spitze des Teleskopmastes ist zum Schutz gegen Blitzschlag noch ein geerdeter Blitzableiter angebracht. Nach der Inbetrieb- 
nahme im April 1995 und der Kalibrierung jedes einzelnen Meßsystems wurde sogleich mit der Aufnahme von Meßdaten begonnen, nur unterbrochen durch die Zeiten, in denen Teile des Systems ausfielen, oder in denen Reparatur- und Wartungsarbeiten an den Systemen oder an Teilen durchgefuihrt werden mußten.

Die Datenverarbeitung und -sicherung geschieht zur Zeit noch im Inselbetrieb innerhalb des Containers, es ist jedoch geplant, wie bereits erwähnt, den Datenzugriff in den Gebäuden der TFH Wildau zu ermöglichen und den Anschluß an die Immissionsmeßnetze der Bundesländer Berlin und Brandenburg herzustellen.

\section{Meßsysteme des Containers}

Es wurden Standardsysteme erworben, die auch in den Bundesländern Berlin und Brandenburg eingesetzt werden.

Alle Meßsysteme haben Komponenten, die sich außerhalb und die sich innerhalb des Meßcontainers befinden. Das Ansaug- und Probenahmerohr für die gasförmigen Luftschadstoffe ist etwa 2,75 m lang mit einem Innendurchmesser von $2,5 \mathrm{~cm}$, durch das ein stetiger Außenluftstrom von 1,824 Kubikmetern pro Stunde $\left(\mathrm{m}^{3 /} / \mathrm{h}\right)$ gesogen wird. Die äußere Ummantelung dieses Rohres besteht außerhalb des Containers aus einer Edelstahl- legierung, mit einer Abschirmung nach oben, die verhindern soll, daß größere Teile wie z. B. Blätter oder Verschmutzungen oder der Kot von Vögeln in das System gelangen können. Das Rohrinnere besteht aus Borosilikatglas mit verschiedenen weiteren Öffnungen, an denen aus dem kontinuierlichen Außenluftstrom Nebenluftströme für die verschiedenen Immissionsmeßgeräte abgezweigt werden können.

Es werden die Gase Kohlenmonoxid (CO), Ozon (O3), Schwefeldioxid (SO2) und die Stickstoffoxide (NO, NO2 = NOX) gemessen. Jedes Meßgerät verfügt uiber eine Pumpe, die dem kontinuierlichen Gasstrom die für die Messungen benötigten Luftmengen fuir jedes der Meßgeräte entnimmt.

Das Ansaug- und Probenahmerohr für die staubförmigen Luftschadstoffe ist etwa 2,95 m lang mit einem Innendurchmesser von $2,5 \mathrm{~cm}$, durch das ein stetiger Außenluftstrom von $1,05 \mathrm{~m}^{3} / \mathrm{h}$ gesogen wird. Dieses Ansaugrohr besteht aus einer Edelstahllegierung, das ebenfalls mit einer Abschirmung nach oben versehen ist. Im Innern des Containers führt dieses Rohr direkt in das Staubmeßgerät, wo der Schwebstaub messend verfolgt wird.

Das Meßgerät verfügt über eine externe Pumpe, die den kontinuierlichen Außenluftstrom uiber ein Filterband leitet. Der auf dem Filter abgeschiedene Staub wird für die Messungen benötigt.

\begin{tabular}{|c|c|c|c|c|c|}
\hline Geräte & Firma & Typ & Meßgröße & Meßbereich & Rauschen \\
\hline $\begin{array}{l}\text { Datenverarbeitung } \\
\text { Computer } \\
\text { Datalogger } \\
\text { Monitor }\end{array}$ & $\begin{array}{l}\text { Horiba Europe GmbH } \\
\text { Hitachi }\end{array}$ & $\begin{array}{c}\text { WCT } \\
\text { Gemi IO } 70151 \\
14 \text { MVX Plus }\end{array}$ & - & - & $\begin{array}{l}- \\
-\end{array}$ \\
\hline $\begin{array}{l}\text { Gasmeßsysteme } \\
\mathrm{CO} \text {-Monitor } \\
\mathrm{NO}_{\mathrm{X}} \text {-Monitor } \\
\mathrm{O}_{3} \text {-Monitor } \\
\mathrm{SO}_{2} \text {-Monitor }\end{array}$ & $\begin{array}{l}\text { Horiba Europe } \mathrm{GmbH} \\
\text { Horiba Europe GmbH } \\
\text { Horiba Europe GmbH } \\
\text { Horiba Europe GmbH }\end{array}$ & $\begin{array}{l}\text { APMA 350E } \\
\text { APNA } 300 \\
\text { APOA } 300 \\
\text { APSA } 350 E\end{array}$ & $\begin{array}{l}\text { Kohlenmonoxid } \\
\text { Stickstoffoxide } \\
\text { Ozon } \\
\text { Schwefeldioxid }\end{array}$ & $\mid \begin{array}{r}0,07-116 \mathrm{mg} \text { pro } \mathrm{m}^{3} \\
0,008-3,8 \mathrm{mg} \text { pro } \mathrm{m}^{3} \\
0,02-4,0 \mathrm{mg} \text { pro } \mathrm{m}^{3} \\
0,003-27 \mathrm{mg} \text { pro } \mathrm{m}^{3}\end{array}$ & $\begin{array}{r}0,03 \mathrm{mg} \text { pro } \mathrm{m}^{3} \\
0,004 \mathrm{mg} \text { pro } \mathrm{m}^{3} \\
0,010 \mathrm{mg} \text { pro } \mathrm{m}^{3} \\
0,0013 \mathrm{mg} \text { pro } \mathrm{m}^{3}\end{array}$ \\
\hline $\begin{array}{l}\text { Staubmeßsystem } \\
\text { Staubmeßgerät }\end{array}$ & $\begin{array}{l}\text { FAG Kugelfischer } \\
\text { Georg Schäfer }\end{array}$ & FH 62 IT & Schwebstaub & $0,01-4,8 \mathrm{mg}$ pro $\mathrm{m}^{3}$ & $0,005 \mathrm{mg}$ pro $\mathrm{m}^{3}$ \\
\hline $\begin{array}{l}\text { Äußere Gas- und Staub- } \\
\text { probennahmesysteme }\end{array}$ & Horiba Europe GmbH & - & - & - & 一 \\
\hline $\begin{array}{l}\text { Metereologische Meßstation } \\
\text { Blitzschutzstab } \\
\text { Geberelektronik } \\
\text { Hygro-Thermogeber } \\
\text { Kombinierter Windgeber } \\
\text { Niederschlagsgeber } \\
\text { Pyranometer } \\
\text { Teleskopmast } \\
\text { Wetter- und Strahlenschutz }\end{array}$ & $\begin{array}{l}\text { Adolf Thies GmbH \& Co KG } \\
\text { Adolf Thies GmbH \& Co KG } \\
\text { Adolf Thies GmbH \& Co KG } \\
\text { Adolf Thies GmbH \& Co KG } \\
\text { Adolf Thies GmbH \& Co KG } \\
\text { Adolf Thies GmbH \& Co KG } \\
\text { Adolf Thies GmbH \& Co KG } \\
\text { Adolf Thies GmbH \& Co KG }\end{array}$ & $\begin{array}{l}- \\
- \\
- \\
- \\
\mathrm{CM} 11 \\
- \\
-\end{array}$ & $\begin{array}{c}- \\
\text { - } \\
\text { relative Feuchte } \\
\text { und Temperatur } \\
\text { Windgeschwindigkeit } \\
\text { Niederschlag } \\
\text { Strahlungsfluß } \\
\text { - } \\
\text { - }\end{array}$ & $\begin{array}{c}- \\
- \\
2-100 \% \\
-35 \text { bis }+70^{\circ} \mathrm{C} \\
0-360^{\circ} \\
0,1-5,5 \mathrm{~mm} \text { pro min } \\
2-1400 \mathrm{~W} / \mathrm{m}^{2} \\
-\end{array}$ & $\begin{array}{c}- \\
- \\
2 \% \\
0,3^{\circ} \mathrm{C} \\
2,5^{\circ} \\
0,1 \mathrm{~mm} \text { pro } \mathrm{min} \\
2 \mathrm{~W} / \mathrm{m}^{2} \\
- \\
-\end{array}$ \\
\hline Klimasystem & York International GmbH & MHC/MOC 12 & Kälteleistung & $3,5 \mathrm{~kW}$ & - \\
\hline $\begin{array}{l}\text { Bislang nicht integrierte } \\
\text { Meßgeräte } \\
\text { Flammenionisationsdetektor } \\
\text { (FID) }\end{array}$ & Bernath Atomic GmbH & Modell 3005 & $\begin{array}{c}\text { Gesamtkohlen- } \\
\text { wasserstoff }\end{array}$ & $\begin{array}{c}10-100.000 \mathrm{ppm} \\
\text { substanzabhängig, be- } \\
\text { zogen auf Propan } \mathrm{C}_{3} \mathrm{H}_{8}\end{array}$ & $\begin{array}{r}<1,5^{\circ} \text { vom Meß- } \\
\text { bereichendwert }\end{array}$ \\
\hline
\end{tabular}

Tab. 1: Überblick über die wichtigsten Parameter der Kontroll-, Meß- und Probenahmesysteme des Containers 
Auf einem Teleskopmast von etwa $6 \mathrm{~m}$ Höhe sind an der Spitze die Windgeschwindigkeits- und Windrichtungsmeßgeräte mit den entsprechenden Sensoren montiert. In etwa einem Meter Höhe iiber dem Dach des Containers ist am Teleskopmast ein Quermast befestigt, auf dem sich das Pyranometer und der Hygro-Thermogeber mit der äußeren Elektronik befinden. Das Pyranometer ermittelt die Globalstrahlung, das Hygrometer die relative Luftfeuchte und das Thermometer die Lufttemperatur: Zum Schutz des Hygro-Thermogebers befindet sich daruiber ein Wetter- und Strahlungsschutz aus Messing und eloxiertem Aluminium.

Der Niederschlagsgeber zur Messung von Regen, Schnee und von Tau befindet sich etwa in einem Meter Höhe über dem Erdboden mit den zugehörigen Meßsonden. Eine eigene Steuerelektronik im Containerinnern verbindet diese meteorologischen Meßgeräte.

Die Systeme zur Regulierung der Containerraumtemperatur bestehen aus einer gewöhnlichen elektrischen Heizung fuir die kalte Jahreszeit und einem Kiihlaggregat für sommerliche Wärmeperioden, das innerhalb des Containers unter der Decke angebracht ist und mit der Wärmeabfuhr auf dem Containerdach gekoppelt ist. Eine wirkungsvollere Containerisolierung wurde bislang nicht angebracht.

\section{Datenaufnahme, -verarbeitung und -transfer}

Bislang wurde eine simultane Datenaufnahme und -verarbeitung vorausgesetzt, tatsächlich sind aber die Orte der Messungen der Luftparameter von denen der meteorologischen Daten und denen der Staubmessungen örtlich bis zu einigen Metern und damit auch zeitlich verschoben. Beschrieben wurden schon die unterschiedlichen Orte, an denen die Daten und die Proben genommen werden.

Die fuir die Gasmessungen angesaugte Luft benötigt einige Zeit, ehe sie von außen kommend an der Abzweigung des Ansaugrohrs ankommt; diese Abzweigungen befinden sich in unterschiedlichen Höhen des Rohres. Von dieser Abzweigung benötigt das Gas wieder Zeit, ehe die Meßzelle erreicht wird, die mit ihrer Volumengröße eine gewisse Luftmenge benötigt, damit das zu messende Gas in der Zelle vollständig erneuert ist.

Der Lufttransport zu den Meßzellen benötigt die Transportzeit $\mathrm{t}_{\mathrm{T}}$, um das Gas in der Meßküvette zu erneuern und die eigentliche Meßzeit $t_{M}$, die das Meßgerät benötigt, um die Messung auszuführen.

Die Luftmeßgeräte und die meteorologischen Meßgeräte produzieren alle drei Sekunden ein Signal, daß über den Datalogger (DAS 1200) auf den Rechner mit dem Immissionsdatenerfassungs- und -auswertungsprogramm (Ida 720) der Firma Horiba uibertragen wird und auf dem Monitor des Rechners abgerufen werden kann. Diese Dreisekundendaten werden arithmetisch fuir jedes Meßgerät gemittelt und durch weitere Mittelungen zu Drei-, Dreißig- und Sechzigminutendaten zusammengefaßt. Eine Sechsstundenmittelung ist ebenfalls möglich. Die Dreiminutendaten werden einen Monat, die höher aggregierten Daten ein Jahr lang gespeichert, ehe sie aus Kapazitätsgründen des Rechners automatisch gelöscht werden. Als Druck können alle Daten ab den Dreiminutendaten als Graphik oder als Tabelle ausgegeben werden.

In den Gebäuden der TFH Wildau existiert ein weiterer Rechner mit Peripherie, auf dem die anfallenden Meßdaten weiter bearbeitet und auf verschiedene Weise graphisch und tabellarisch dargestellt werden können. Dieser Rechner betrifft nicht direkt die Datenerfassung der Containermeßsysteme; die Beschreibung des externen Rechnersystems wird einem späteren Zeitpunkt vorbehalten bleiben.

\section{Die wichtigsten verwandten Meßprinzipien}

Das Kohlenmonoxidmeßgerät basiert auf der spektralen Infrarotabsorption des CO's. Durch eine Meßkiivette wird abwechselnd das Meßgas und das Referenzgas, welches die im Gerät katalytisch CO-befreite Außenluft ist, geleitet. Die Absorption wird mit einem infrarotsensitiven Detektor ermittelt und in Signale verwandelt. Gewisse korrodierende Gase wie SO2, Cl2, F2 oder $\mathrm{HCl}$ beeinträchtigen die Leistungsfähigkeit dieses, wie auch aller anderen Meßgeräte. Das Meßgerät hat eine eigene Pumpe zur Gasversorgung des Systems; regelmäßige Überprüfungen der verschiedenen Aggregate liegen, wie bei allen anderen Meßgeräten, in Zeiträumen von Wochen bis Monaten.

Das Ozonmeßgerät basiert auf der spektralen Ultraviolettabsorption des O3's. Das zu messende Gas wird über zwei Gaswege in die Meßzelle bzw. in die Referenzmeßzelle geleitet und gleichzeitig gemessen, dabei wird dem Referenzgas durch einen O3-Vernichter alles Ozon entzogen. Die Absorptionen werden mittels Photomultiplier gemessen und in Signale verwandelt.

Das Schwefeldioxidmeßgerät basiert auf einer spektralen Ultraviolettanregung des SO2's, welches fluoreszierend die aufgenommene Lichtenergie im Ultravioletten wieder reemittiert.

Das zu messende Gas wird in die Meßzelle geleitet und in zueinander senkrechten Strahlengängen als Erregerlicht und Fluoreszenzlicht gleichzeitig gemessen. Die Absorptionen werden mittels Photomultiplier ermittelt und in Signale verwandelt. Außer SO2 fluoreszieren auch Aromaten, dem Gerät ist daher vor der Meßzelle ein zweistufiger Kohlenwasserstoffabscheider vorgeschaltet. Das Schwebstaubmeßgerät scheidet auf einem Glasfaserfilterband kontinuierlich den Staub der Außenluft ab. Die Messung des Staubbelages des Bandes erfolgt durch Absorption der von dem Radionuklid Krypton (Kr-85) emittierten Elektronen (Betastrahlung) im Vergleich mit den Meßwerten einer Referenzmeßstrecke. Die Meßwerte ergeben sich aus den Änderungen der Strommessungen der lonisationsmeßkammern, die die Signale erzeugen. Die hochenergetischen Elektronen regen auch noch Röntgenniveaus der im Staub vorhandenen Atome an und entsprechend besteht prinzipiell die Möglichkeit, über Röntgenfluoreszenz Aussagen über die Art und die Menge der Atome des angesaugten Staubes zu machen. 
Das Stickstoffoxidmeßgerät basiert auf der Messung von Chemilumineszenzreaktionen der NOX's, der Summe von NO und NO2. NO reagiert mit Ozon zu NO2 und NO2 emittiert beim Übergang vom angeregten in den Grundzustand typisches Chemilumineszenzlicht, welches mit einem Photomultiplier nachgewiesen und zu einem Signal verarbeitet wird. Das bereits vorhandene nichtangeregte NO2 muß vor der Messung in NO umgewandelt werden, ehe die bereits beschriebene Meßmethode angewandt werden kann.

Alle Luftmeßgeräte wurden werksseitig von den Firmen FAG Kugelfischer Georg Schäfer bzw. Horiba eingestellt; die Kalibrierungen der Einzelkomponenten können zur Zeit mit eigenen Standardverfahren noch nicht vorgenommen werden, aber es gibt Hilfezusagen der Leiter der Meßstellen in den Ländern Berlin und Brandenburg. Alle Meßgeräte der meteorologischen Meßstation sind Standardmeßinstrumente, die auch vom Deutschen Wetterdienst eingesetzt werden. Die Außenteile sind aus korrosionsbeständigen Werkstoffen gefertigt und durch Lack geschiitzt. Die meteorologische Meßstation wurde ebenfalls von der Firma Horiba installiert und in das Datenaufnahme und -verarbeitungssystem des Meßcontainers integriert. Alle äußeren Bauteile sind im Winterbetrieb elektronisch geregelt heizbar, um ein Einfrieren zu verhindern.

Der kombinierte Windgeber erlaubt über einen dreiarmigen trägheitsarmen Schalenstern eine berührungslose optische Abtastung der Anemometerdrehzahl und damit der Windgeschwindigkeit in Form von Pulsfrequenzveränderungen. Die Windrichtung wird über eine trägheitsarme Leichtmetallwindfahne erfaßt, die mit einer Codescheibe gekoppelt, optoelektronisch iiber 360 Grad abgelesen wird.

Der Hygro-Thermogeber gibt die Lufttemperatur und die relative Luftfeuchte an. Die Feuchtemessung erfolgt mit einem Haar- oder Kunststoffmeßelement, das aus mehreren Haaren oder Fasern besteht und die entsprechend der Feuchtigkeit ihre Länge ändern. Diese Längenänderung dreht ein Potentiometer, so daß Spannungsanzeigen erfolgen können. Die Lufttemperatur wird mittels eines Platinwiderstandthermometers gemessen, bei dem der Widerstand temperaturabhängig ist.

Die Globalstrahlung wird mit dem Pyranometer durch Reflexion erfaßt, diese ergibt sich aus der direkten Sonneneinstrahlung und der diffusen Streustrahlung des Himmels. Die Lichtenergie, die durch die Geräteglas- halbkugel einfällt, wird durch Absorption in Wärme verwandelt, die als Widerstandsänderung gemessen wird. Über Abdeckungen kann die diffuse von der direkten Strahlung unterschieden werden. Zu Parametern wie Bestrahlungsstärke, Temperatur, etc. gibt es Querkorrelationen, die standortspezifisch ermittelt werden müssen.

Der Niederschlagsgeber mißt den auf die Erdoberfläche fallenden Niederschlag, indem dieser uiber ein Sieb auf eine Kippwaage geleitet wird, die sich bei bestimmten aufgefangenen Wassermengen entleert. Die Kippvorgänge werden als Impulse gezählt und stellen ein Maß für die aufgefangene Niederschlagsmenge dar.

Der bislang noch nicht im Container integrierte Flammenionisationsdetektor (FID) arbeitet vergleichend, es werden die unbekannten organischen Luftkomponenten als Summenwert mit der bekannten Konzentration des Meßgases Propan verglichen. Der FID, in dem eine reine Wasserstoffgas-(H2)-Flamme brennt, ionisiert kohlenstoffhaltige Gase; das angelegte elektrische Feld erzeugt daraus einen Ionenstrom, der der Datenverarbeitung zugänglich ist.

\section{Langzeitmessungen und Systemzuverlässigkeit}

Der Luftmeßcontainer wurde im Frühsommer 1995 mit den Meßgeräten für die luftfremden Stoffe CO, NOX, O3, Staub und $\mathrm{SO} 2$ in Betrieb genommen und war bis Ende 1995 über 6000 Stunden in Betrieb. Die folgende Abbildung 2 und die Tabelle 2 gibt die Ausfallzeiten der einzelnen Meßsysteme und die Gründe dafür an.

Der Abbildung und der Tabelle ist zu entnehmen, daß, abgesehen vom NOX-Monitor, die Ausfallzeiten durch den Rechner verursacht wurden. Die Meßsysteme des

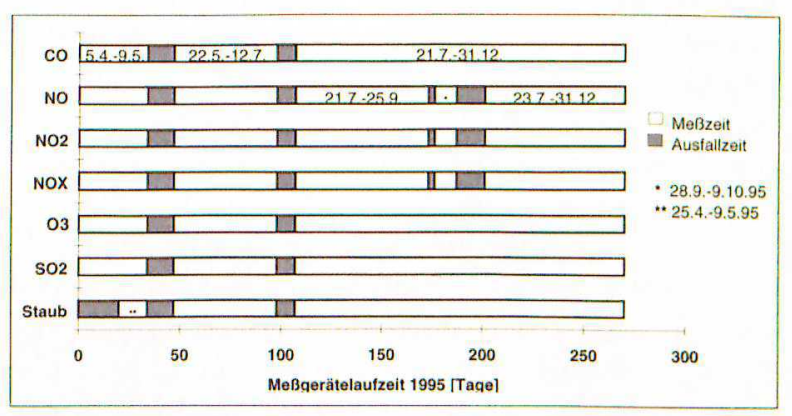

Abb. 2: Ausfall- und Meßzeiten der Luftmeßgeräte des Containers und des Rechners 1995

\begin{tabular}{|l|c|c|c|c|l|}
\hline System & Inbetriebnahme & $\begin{array}{c}\text { Jahresstunden } \\
1995\end{array}$ & $\begin{array}{c}\text { Ausfall 1995 } \\
\text { (in Stunden) }\end{array}$ & (in Prozent) & Ursachen \\
\hline $\begin{array}{l}\text { Gasmeßsysteme } \\
\text { CO-Monitor }\end{array}$ & 5. April 1995 & 6504 & 576 & $8,8 \%$ & Rechnerausfälle \\
NO-Monitor & 5. April 1995 & 6504 & 1008 & $15,5 \%$ & Pumpendefekt und Rechnerausfälle \\
NO2-Monitor & 5. April 1995 & 6504 & 1008 & $15,5 \%$ & Pumpendefekt und Rechnerausfälle \\
NOX-Monitor & 5. April 1995 & 6504 & 1008 & $15,5 \%$ & Pumpendefekt und Rechnerausfälle \\
O3-Monitor & 5. April 1995 & 6504 & 576 & $8,8 \%$ & Rechnerausfälle \\
SO2-Monitor & 5. April 1995 & 6504 & 576 & $8,8 \%$ & Rechnerausfälle \\
\hline Staubmeßsystem & & & & & \\
Staubmeßgerät & 25. April 1995 & 6024 & 576 & $9,6 \%$ & Rechnerausfälle und spätere Inbetriebnahme \\
\hline
\end{tabular}

Tab. 2: Überblick über die Systemzuverlässigkeit der Kontroll-, Meß- und Probenahmesysteme des Containers 1995 
Containers werden an zwei Tagen in der Woche betreut und gewartet. Fällt zum Beispiel das elektrische Netz nach diesen Arbeitstagen kurzzeitig aus oder gibt es Netzschwankungen, die den Rechner abschalten, werden Meßdaten produziert, aber nicht gespeichert.

Im NOX-Monitor zeigte die Pumpe Defekte und mußte nach einem Reparaturversuch durch eine neue Pumpe ersetzt werden. Hinsichtlich der Datenaufnahme bedeutet dies den Verlust von NOX-Datenreihen, weil - sofern der Rechner nicht ebenfalls abschaltet oder defekt ist die anderen Systeme weiterhin Meßwerte produzieren und diese gespeichert werden.

Die Meßsysteme der meteorologischen Station sollten im Spätherbst 1995 in Betrieb gehen. Der Aufbau der äußeren Teile dieses Meßsystems hat sich jedoch aus verschiedenen Gründen so verzögert, daß dann auch wegen des frühen und außerordentlich langen und kalten Winters die Inbetriebnahme bis in das Jahr 1996 verschoben werden mußte. Die meteorologischen Meßdaten werden an dieser Stelle noch nicht angegeben und ausgewertet.

\section{Zwei ausgewählte Meßepisoden}

Die folgenden Abbildungen 3 bis 7 zeigen, welche Informationen uiber CO, O3, NOX, SO2 und Staub im Rechner gespeichert werden. Eine weitergehende Interpretation verbietet sich an dieser Stelle, da einige Untersuchungen zur Zuverlässigkeit des Systems noch durchzufuihren sind.

Standardmäßig werden regelmäßig die Tages-, Wochenund Monatswerte uiberwacht. Die regelmäßige Datenausgabe erfolgt wöchentlich und monatlich, und die Werte werden graphisch ausgegeben in der Technischen Fachhochschule zum Aushang gebracht.

An Hand der Abbildungen soll versucht werden, einige Zusammenhänge zwischen den gemessenen Luftparametern zu verdeutlichen. Weitergehende Interpretationen, insbesondere die Zuordnung zwischen Emittenten, Transportwegen der Luftschadstoffe, luftchemischen Reaktionen und den gemessenen Immissionen bleiben späteren Veröffentlichungen vorbehalten.

Bekannt ist, daß bei starker Sonneneinstrahlung und vorhandenen weiteren luftverunreinigenden Stoffen der Ozonanteil in der Atmosphäre zunimmt, dies ist den Monatstrends fuir August und Dezember 1995 zu entnehmen.

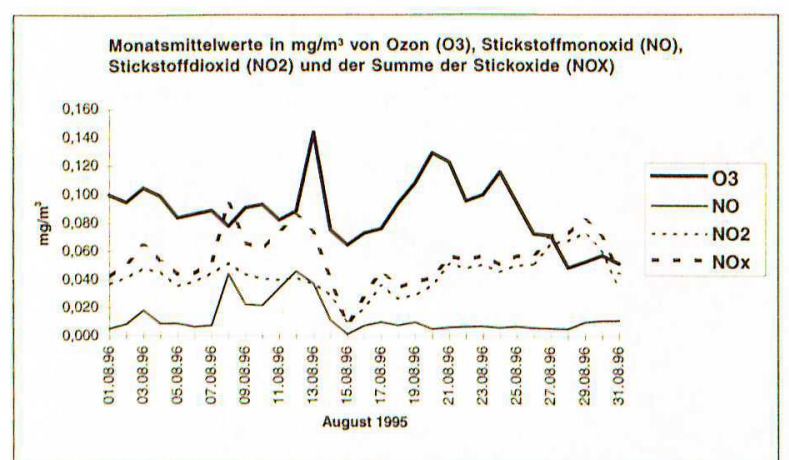

Abb. 3: Monatstrends von 03, NO und von NOX vom August 1995 am Standort TFH Wildau

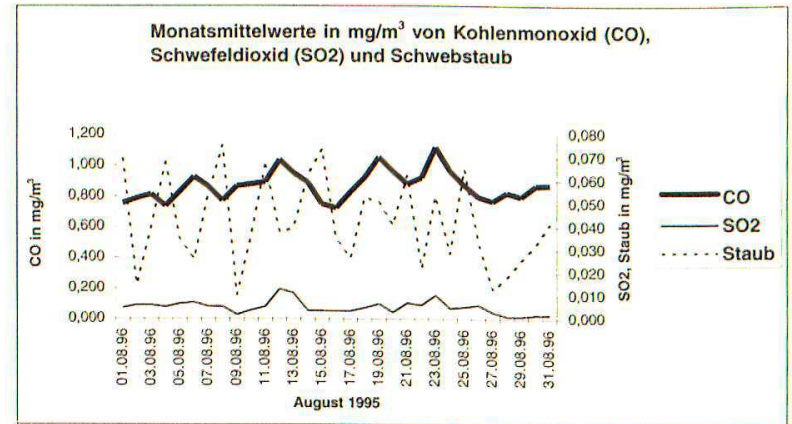

Abb. 4: Monatstrends von CO, SO2 und Staub vom August 1995 am Standort TFH Wildau

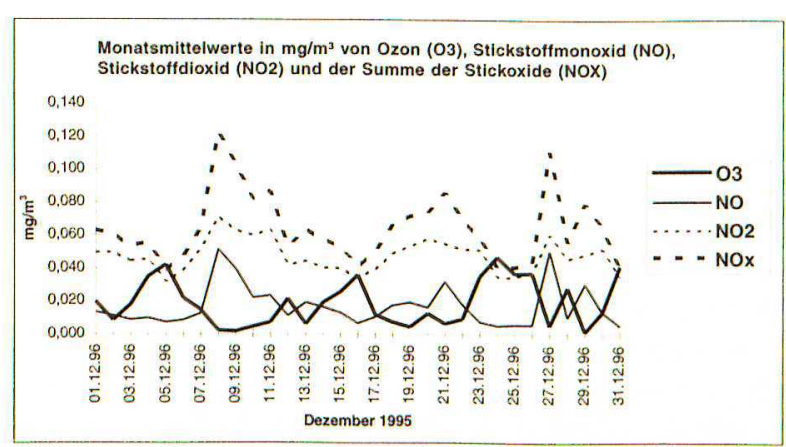

Abb. 5: Monatstrends von 03, NO, NO2 und von NOX vom Dezember 1995 am Standort TFH Wildau

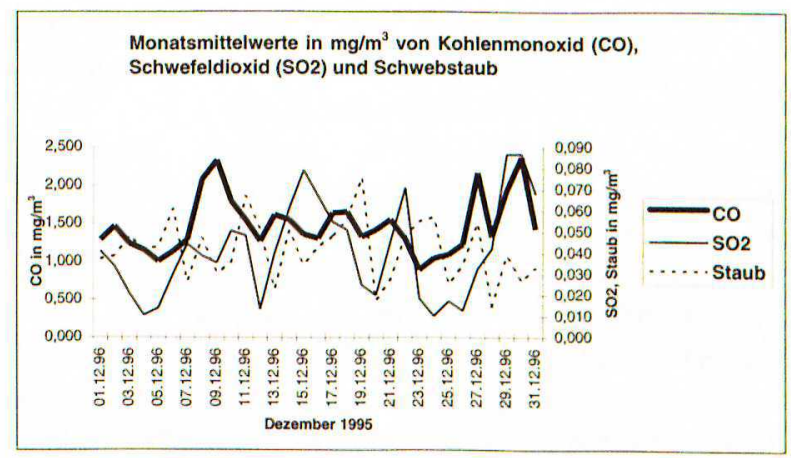

Abb. 6: Monatstrends von CO, SO2 und Staub vom Dezember 1995 am Standort TFH Wildau

Im Dezember 1995 ist der Ozonanteil der bodennahen Luft wesentlich geringer als im August. Die NOX-Anteile sind im Vergleich zum August nur geringfügig erhöht, während die $\mathrm{CO}$ - und SO2-Anteile eine sprunghafte $\mathrm{Zu}$ nahme zeigen. Die Staubanteile in der Luft unterscheiden sich in den Monaten August und Dezember 1995 jedoch kaum voneinander.

Die fallende Außentemperatur im Dezember vermehrt den Hausbrand, damit steigen die Oxidationsraten des Kohlenstoffs fossiler Brennstoffe zu CO, des Schwefels in fossilen Brennstoffen zu SO2 und des Luftstickstoffs N2 zu Stickoxiden NOX. Obwohl die Gesamtbelastung der bodennahen Atmosphäre in den Wintermonaten größer als in den Sommermonaten ist, zeigt der Ozontrend im Dezember geringere Werte als im August 1995, weil die Intensität des eingestrahlten Sonnenlichtes und damit die Photoproduktion des Ozons gering ist.

Die Abbildung 7 greift aus dem Monatstrend des August 1995 einen Tag heraus, an dem die Ozonwerte in Wildau ein Maximum erreichten. Besonders hohe 03-Werte 


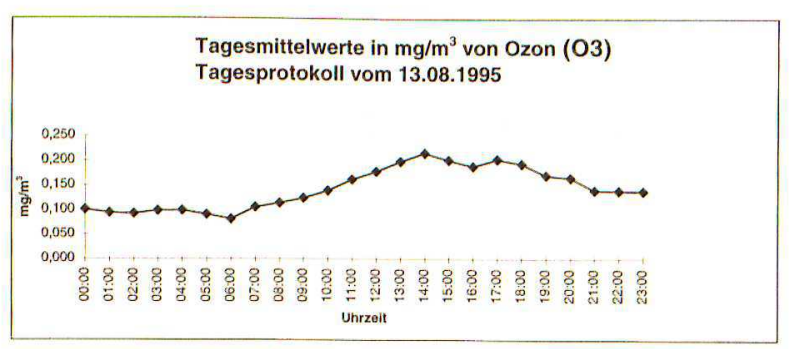

Abb. 7: Maximum des Tagestrends von Ozon vom 13. August $1995 \mathrm{am}$ Standort TFH Wildau als Stundenwerte

werden am 13. August gemessen. Mit zunehmender Uhrzeit und damit Sonneneinstrahlung wird das Maximum der O3-Werte mit einem Wert von 200 Mikrogramm pro Kubikmeter zwischen 13 und $14 \mathrm{Uhr}$ erreicht, ein Meßwert, der oberhalb empfohlener Grenzwerte liegt.

Bislang sind direkte Zuordnungen zu entsprechenden Emittenten nicht versucht worden und nicht durchführbar gewesen, da 1995 der Aufbau und die Inbetriebnahme der meteorologischen Station noch nicht erfolgt war und der laufende Meßbetrieb weitergehende Untersuchungen in dieser Phase des Aufbaus erschwerten.

Es ist aber bekannt, daß zur lokalen Belastung der bodennahen Luft auch großräumige Emissionen beitragen. Diese ließen sich in der Tendenz dahingehend ablesen, daß in den Wintermonaten langanhaltende Ostwindperioden andere Schadstofftrends in der Atmosphäre produzieren als Nord- oder Westwindperioden.

\section{Ausblick}

Der Luftmeßcontainer der TFH Wildau enthält hochwertige Meßgeräte, die es erlauben, den Studenten einige wichtige Immissionsmeßtechniken der Luftumgebungsuiberwachung und deren Datenverarbeitung in ihrer Ausbildung zur Kenntnis zu bringen. Gleichwohl sind Ergänzungen und Verbesserungen an den Systemen wïnschenswert, etwa von der folgenden Art: die Kalibrierung der Meßgeräte sollte in regelmäßig wiederkehrenden Zeiträumen stattfinden, anfangs über die Betreiber des Berliner oder des brandenburgischen Meßnetzes, später durch den Aufbau von Eichverfahren zum Beispiel als Vergabe von Diplom- oder Studienarbeiten in der TFH Wildau nach den Standardverfahren des Vereins deutscher Ingenieure (VDI).

Eine zügige Ankopplung der Systeme des Luftmeßcontainers an die Datenverarbeitung im Gebäude der TFH Wildau und an den Fachbereich Physikalische Technik und an deren Labore wäre wünschenswert, und danach sollte es möglich sein, mit den Landesmeßnetzen Berlins und Brandenburgs in einen Datentransfer einzutreten.

Weiterhin könnte iiberlegt werden, zum Teil bereits in der TFH Wildau vorhandene Meßgeräte wie den Flammenionisationsdetektor in das Meßsystem zu integrieren, auch ließen sich einige Meßgeräte in ihren Meßmöglichkeiten ergänzen.

In der Zukunft werden sicherlich die klassischen luftgetragenen Schadstoffe auch in den neuen Bundesländern in ihrer Bedeutung abnehmen, sofern diese Schad- stoffe nicht uiber große Entfernungen einen grenzuiberschreitenden Import darstellen. Damit eröffnen sich für die TFH Wildau Möglichkeiten, die Empfindlichkeiten der vorhandenen Meßgeräte zu steigern oder/und weitere Meßgeräte mit anderen naturwissenschaftlichen Meßprinzipien zu beschaffen oder an der TFH Wildau selbst zu entwickeln und einzusetzen.

\section{Verfasser}

Prof. Dr. rer. nat. Klaus R. F. Bätjer Iris Geike

Technische Fachhochschule Wildau Fachbereich Physikalische Technik Tel. (0 33 75) 507-121, -194 